\title{
COMPARISONS BETWEEN RESULTS OF POLAR COORDINATES DERIVED FROM TIME DATA AND THOSE FROM LATITUDE ONES
}

\author{
SEICHI OKAZAKI and MITSUKO NASAKA \\ Tokyo Astronomical Obser vatory, Japan
}

\begin{abstract}
Computations of the polar coordinates are carried out by the use of time data and of latitude data separately. The results from time data and those from latitude data are proved to have mutual differences in annual term in particular. That is, polar loci obtained from latitude results give almost circular forms, while those from time results give elliptic forms elongated rather seriously on the $X$-axis.
\end{abstract}

In this paper instantaneous polar coordinates are computed from data of latitude observations and from those of time observations independently, and the results are compared with each other.

The data of time and latitude observations made with PZT at eight observatories (Mizusawa, Tokyo, Neuchâtel, Greenwich, Ottawa, Washington, Richmond, and Mount Stromlo) and those with Danjon astrolabes at three observatories (Potsdam, Alger, and Paris) during the period of 6 yr from 1962 to 1967 are used here. The eleven observatories are located mainly in three directions in longitude. Computations have been made combining the data of all eleven observatories and also by combining those of a selected nine observatories and a selected seven.

The results obtained from latitude data, $\left(x_{\varphi}, y_{\varphi}\right)$, and those from time data, $\left(x_{\tau}, y_{\tau}\right)$, are proved to have mutual differences in periodic and secular terms, for any of three combinations. The results averaged for three combinations of eleven, nine and seven observatories are given in Table $\mathrm{I}$, when $x$ and $y$ are expressed in the following form,

$$
a+b t+A \sin \left(2 \pi t+\theta_{1}\right)+B \sin \left((2 \pi / 1.2) t+\theta_{2}\right),
$$

and $t$ is reckoned in fractions of a year from 1965.0.

TABLE I

\begin{tabular}{|c|c|c|c|c|c|c|}
\hline & $a$ & $b$ & $A$ & $\theta_{1}$ & $B$ & $\theta_{2}$ \\
\hline$x_{\varphi}$ & $0 " 001$ & +0.0071 & 0".086 & $194^{\circ}$ & 0"146 & $189^{\circ}$ \\
\hline$x_{\tau}$ & $0 " 009$ & -0.0053 & $0 " 121$ & $225^{\circ}$ & 0 "145 & $189^{\circ}$ \\
\hline$y_{\varphi}$ & $0 " 243$ & -0.0025 & 0".088 & $288^{\circ}$ & 0 "!141 & $281^{\circ}$ \\
\hline$y_{\boldsymbol{\tau}}$ & $0 " 232$ & $-0 ! 0006$ & $0 ! 063$ & $298^{\circ}$ & $0 " 155$ & $283^{\circ}$ \\
\hline
\end{tabular}

As to the annual term, the polar locus drawn with the average values of $\left(x_{\varphi}, y_{\varphi}\right)$ is almost circular in form, and that is similar to the results by the five ILS stations, while the polar locus with $\left(x_{\tau}, y_{\tau}\right)$ is like a slender ellipse, the major axis of which is almost parallel to the $X$-axis. On the other hand, the polar loci of the Chandler term coincide 
with each other without significant difference. The difference in the secular term seems uncertain, because the interval of data utilized here is not sufficient.

Moreover similar results to those mentioned above have been obtained also on the data covering the longer interval of $12 \mathrm{yr}$ from 1958 to 1969. B. Guinot and M. Feissel (1968) published their results derived from data during $2.5 \mathrm{yr}$ from 1964.00 to 1966.45 , to which the present results are quite similar, as for the annual components.

Thus this may suggest that the polar loci of the annual components, the almost circular form for the latitude results and the somewhat slender elliptic form for the time results, are almost free from local errors or the period of data utilized.

\section{References}

Guinot, B. and Feissel, M.: 1968, in Wm. Markowitz and B. Guinot (eds.), 'Continental Drift, Secular Motion of the Pole, and Rotation of the Earth', IAU Symp. 32, 63.

Okazaki, S. and Nasaka, M.: 1971, Ann. Tokyo Astron. Obs. XII, No. 3, 175.

\section{DISCUSSION}

S. Débarbat: How did Mr. Okazaki combine the 11, 9, and 7 observatories? And what were the differences?

S. Okazaki: The 11 observatories are all the stations of which data are utilized in this paper; the 9 observatories involve three stations in each of the three directions in longitude, except Potsdam and Alger; the 7 observatories are selected with the following conditions:

$$
\Sigma \sin \lambda_{i} \fallingdotseq \Sigma \cos \lambda_{i} \fallingdotseq 0,
$$

where $\lambda_{i}$ is the adopted longitude for the $i$-th station. In this case, Greenwich and Washington are further omitted from the 9 observatories. Differences among the results of the three combinations do not show a significant systematic tendency, and the individual differences for every twentieth of a year are $\pm 0.010 \sim \pm 0.025$ in standard deviations, as average.

B. Guinot: Remark that a study recently carried out by Miss M. Feissel confirms the conclusions of Okazaki. 